\title{
Gene Therapy and Vector Shared Resource
}

National Cancer Institute

\section{Source}

National Cancer Institute. Gene Therapy and Vector Shared Resource. NCI Thesaurus. Code C39410.

The Gene Therapy and Vector Shared Resource is dedicated to the construction and production of viral and non-viral vectors to Cancer Center faculty members for use in human preclinical and clinical, and animal gene therapy studies, supports protocol development, study strategy development, and gene therapy adverse event risk assessment. 